\title{
A Study on Smart Home Control System through Speech
}

\author{
Parameshachari B D Sawan Kumar Gopy Gooneshwaree Hurry Tulsirai T. Gopaul \\ Department of Electronics \& Communication Engg., JSS Academy of Technical Education, \\ Avenue Droopnath Ramphul, Bonne Terre, Vacoas, Mauritius.
}

\begin{abstract}
This paper proposes an automation system where users can use voice commands to control their electrical appliances, such as light, fan, television, heater etc. The main aim is to help make the life of people more comfortable, especially for the elderly and disabled as they will not have to be actually present near an appliance to turn it on or off. The system focuses on using a Digital Signal Processor to process the voice commands and accordingly control the required appliance. XBee transceivers are used to eliminate the need for large amount of wiring between the processor and the appliances. Experimental results show that the system has a good response and is cost effective. We conclude that this system provides solutions for the problems faced by home owners in daily life and make their life easier and more comfortable by proposing a cost effective and reliable solution.
\end{abstract}

\section{General Terms}

Home Automation, Centralised Appliance Control

\section{Keywords}

Spoken command, XBee, Zero Crossing, Speech Recognition

\section{INTRODUCTION}

Speech recognition is a vast topic of interest and is looked upon as a complex problem. In a practical sense, speech recognition solves problems, improves productivity, and changes the way we run our lives. Reliable speech recognition is a hard problem, requiring a combination of many techniques; however modern methods have been able to achieve an impressive degree of accuracy [1]. Real-time digital signal processing made considerable advances after the introduction of specialized DSP processors. Suitable DSP Starter Kits, with specific DSP processor and related software tools such as assemblers, simulators and debuggers are available to make system design and application development easier. Digital Signal Processor TMS320C5535 enables to design a system with very high computational power and large memory space with minimal count of components which saves printed circuit board space and simplifies the design $[2$, $3]$.

ZigBee is a low cost and low power wireless mesh network standard. The low-cost allows it to be used for a wireless monitoring and control applications and low power allows longer battery life. Mesh networking provides high reliability and more extensive range. The ZigBee standard operates on the IEEE 802.15.4 physical radio specification and uses unlicensed bands which include $2.4 \mathrm{GHz}, 900 \mathrm{MHz}$ and 868 MHz. ZigBee uses Carrier Sense Multiple Access Collision
Avoidance (CSMA/CA) to increase reliability. Before transmitting, it listens to the channel and when the channel is clear, begins to transmit, preventing corrupted data. ZigBee uses a 16-bit CRC on each packet, called a Frame Checksum (FCS). This ensures that the data bits are correct. Each packet is retried up to three times. If the packet cannot get through after the fourth transmission, ZigBee informs the sending node about it [4]. XBee is the brand name from Digi International for a family of radio modules. The first XBee radios were based on the 802.15.4 standard designed for point-to-point and point-to-multipoint communications at baud rates of $250 \mathrm{kbit} / \mathrm{s}$.

The rest of the paper is organised as follows: section 2 discusses related work to proposed schemes, section 3 committed to proposed framework including the hardware design and the software design along with algorithm. Section 4 discusses results and finally concluding remarks are given in section 5 .

\section{RELATED WORK}

Nguyen et al. [5] proposed a Home appliance control system. Infrared ray and power line communication are used to control the home appliances system. This system helps user to checks the status of appliances and controls them remotely from everywhere. And this is done through their cellular phone or Internet. The simple approach to control the home appliances is given in this paper.

Haque et al. [6] proposed a system that controls the home appliances using the personal computer. This system is developed by using the Visual Basic 6.0 as programming language and Microsoft voice engine tools for speech recognition purpose. Appliances can be either controlled by timer or by voice command.

Khiyal et al. [7] proposed a system for controlling home appliances remotely that is useful for the people who are not at home mostly. The main objective of the system is to provide security and control the home appliances such as AC, lights and alarms. The system is implemented by SMS technology that is used to transfer data from sender to receiver over GSM network. One or more computers can be used to control the home appliances. System send an alert SMS to authorized user when any intrusion is detected and user can in turn respond in order to overcome the situation. Moreover user can send SMS to system to get the status of home appliances and controlling them.

Jawarkar et al. [8] proposed the software system for communication between mobile and computer. UART 16550 A chip is programmed using appropriate control format to support AT command. The mobile in this system is used for receiving and executing commands from preconfigured users 
and informing status about change in input to the user through SMS. The system can also send SMS to specified mobile user if there is a change in the status of the input ports. This system is not for time critical systems.

IPrabhakar V. Mhadse et al. [9] proposed A home automation system based on voice recognition was built and implemented. The prototype developed can control electrical devices in a home or office. The system implements Automatic Speech Recognition using speech processor and MATLAB coding.

S. R. Suralkar et al. [10] A speech recognized automation system using speaker identification through wireless communication is built and implemented. The prototype developed can control electrical devices in a home or office wirelessly. The system implements Automatic Speech Recognition using speech processor and Speaker Identification through MATLAB coding using MFCC algorithm.

\section{PROPOSED METHOD}

The proposed method is aimed at designing a voice controlled smart home control system for the following reasons. One of the major problems in our present day society is wastage of energy, whereby energy consumption is continuously increasing year by year. Nowadays, some people may be too lazy or too busy to get up and turn off a particular appliance. Hence, the smart home control system will be useful as one will only have to speak to turn off a device, thereby saving energy as well as one's time. Moreover, old or disabled persons may experience difficulties in going around the house to turn on/off their appliances, especially if they live alone. It will be much easier for them to use the voice control system. It will also help blind people as they will be able to turn on a fan or a radio without relying on others. The system is designed in such a way that it is easy to install and use. The proposed method is to use a Digital Signal Processor (DSP) for speech processing and recognition. The output of the DSP will be sent, through the XBee transceivers, to the control part, where a microcontroller will select the required device according to the input voice command.

The system can be divided into 3 main parts:

1. Audio processing part $\rightarrow$ microphone and DSP

2. Transmission part $\rightarrow$ XBee transceivers

3. Control part $\rightarrow$ microcontroller and relays

The voice commands will first be captured and processed in the DSP according to the voice recognition method used. After successful identification of the commands, control characters will be wirelessly sent through the XBee transceivers to the microcontroller, which will in turn activate the corresponding relay. As a result, home appliances could be turned on or off depending on the voice command given.

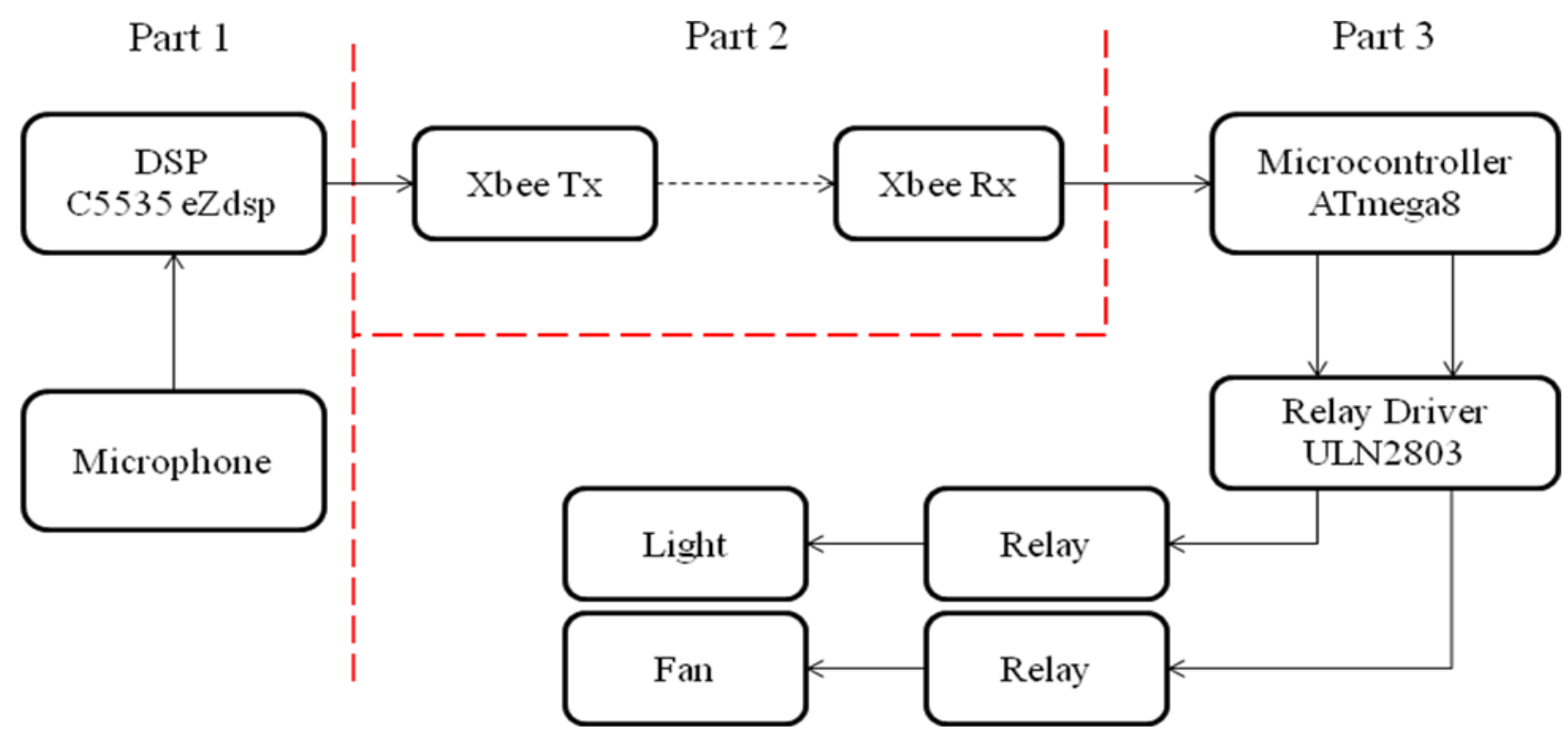

Fig 1: Proposed block diagram of the Smart Home Control System

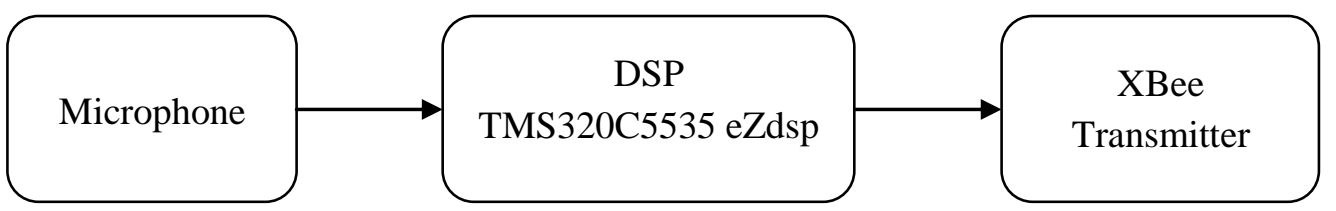

Fig 2: Block diagram of Audio Processing Part

\subsection{Hardware Design}

In this section, we will present the hardware description of the 3 main parts that form our Smart Home Control System.

\subsubsection{Audio Processing}

The first part of the system is as shown in figure 2. The voice is captured by the microphone and given to the 
TMS320C5535 eZdsp for processing so as to differentiate between the voice commands. The speech processing is carried out using the zero crossing with peak amplitude (ZCPA) method. If the required conditions are satisfied, that is, a valid command is recognized, a specific signal will be sent, using UART, to the XBee transmitter and consequently to the control part. Figure 3 shows the DSP board on which voice commands are processed and recognized accordingly. The block diagram of the C5535 eZdsp is shown in Figure 4.
The TMS320C5535 are the lowest cost and lowest power 16bit processors, helping conserve energy and enabling longer battery life. Having a 240 MIPS (million instructions per second) performance, up to $320 \mathrm{~KB}$ on-chip memory, higher integration (including a hardware accelerator for FFT computation), these processors provide a foundation for a range of signal processing applications, including voice and audio processing [11].

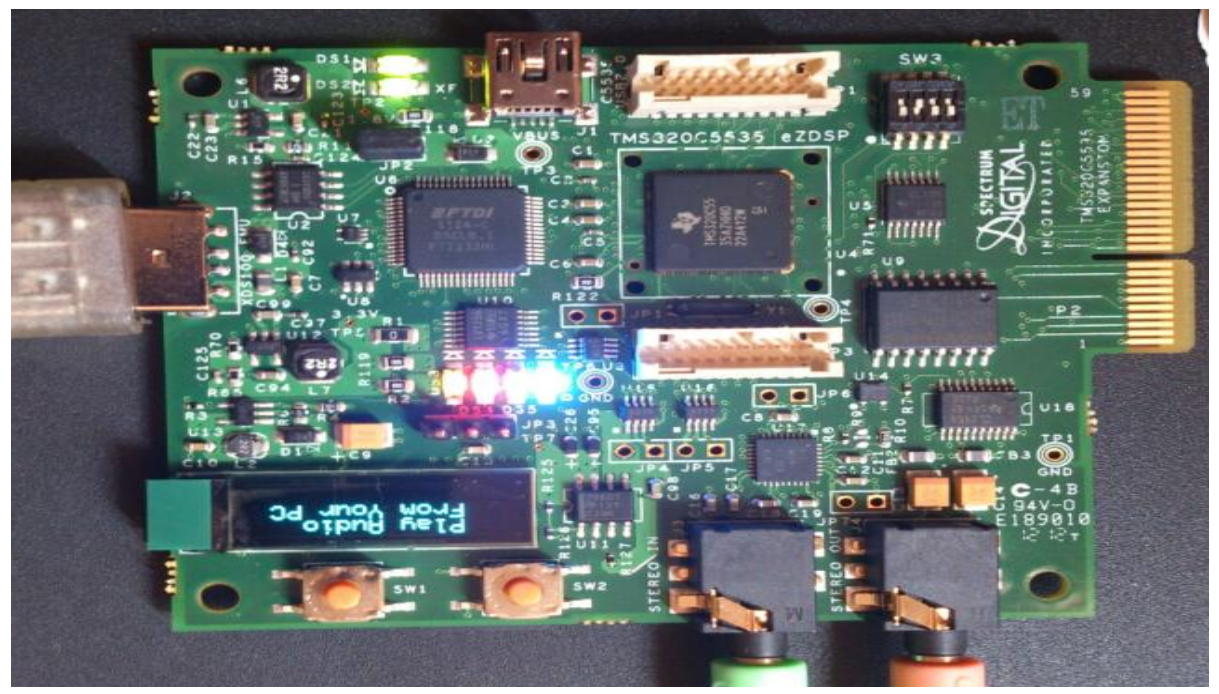

Fig 3: C5535 eZdsp board

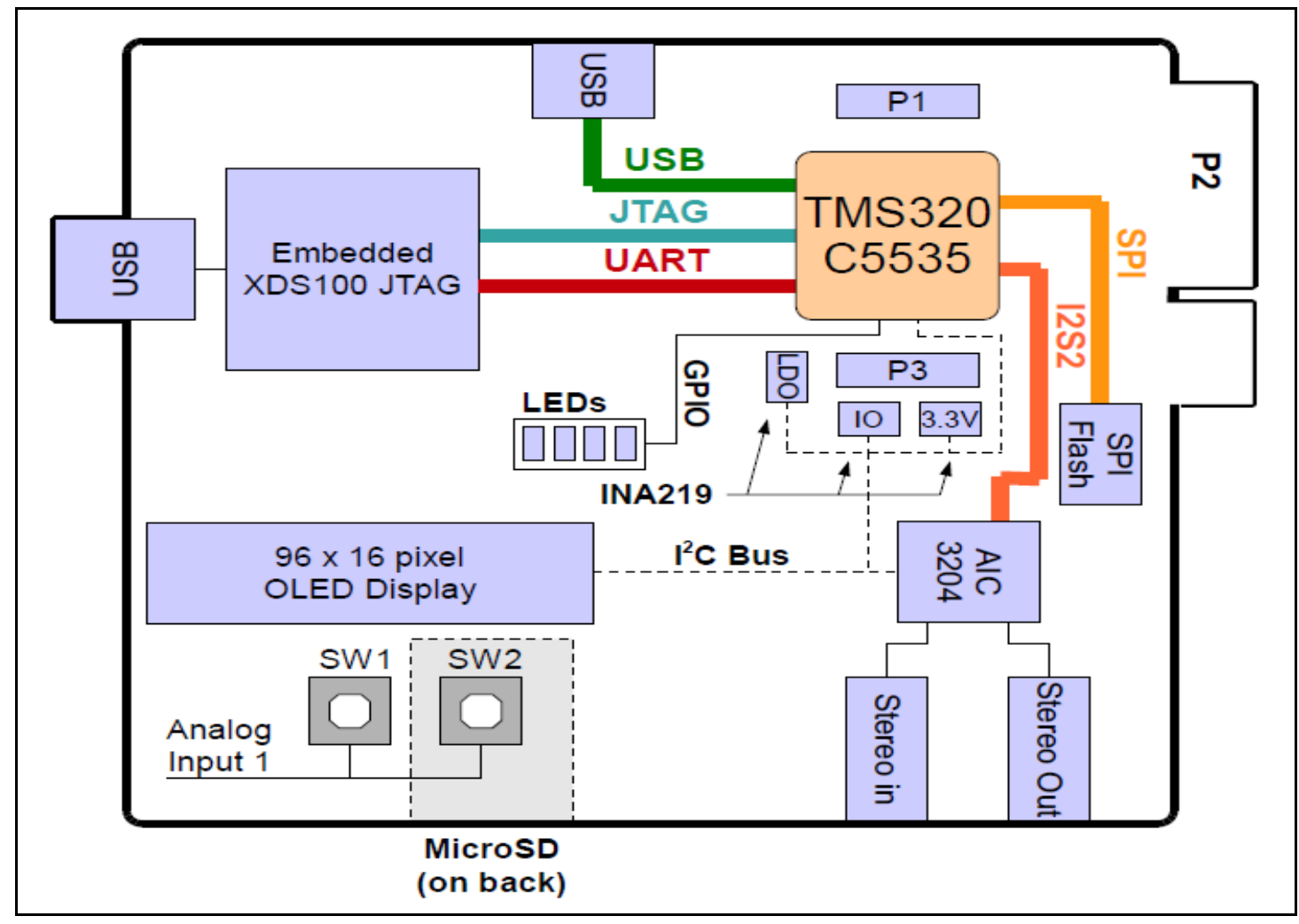

Fig 4: C5535 eZdsp block diagram

\subsubsection{Transmission}

The second part of the project concerns wireless transmission of signals using XBee transceivers. Once voice commands have been recognized, corresponding control characters are sent through the XBee transmitter at a baud rate of 9600 bps. The XBee transceiver and XBee programmer are as shown in figure 5 and 6. 

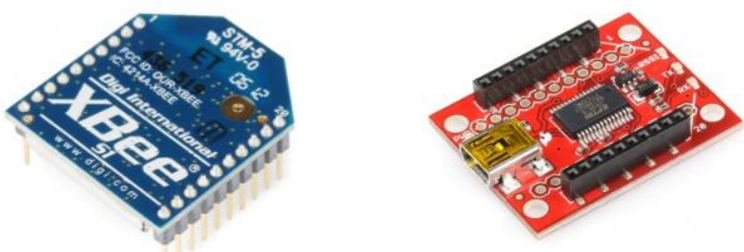

Fig 5: XBee Transceiver
Figure 7. A range test and loopback test were carried out to test the functioning of the XBee modules. The results are shown in Figures 8 and 9 below.

Transmitted data is shown in blue colour and received data in red colour in the loopback test. In the range test, the software will repeatedly send packets of data and display the signal strength as well as the percentage of correctly returned packets. RSSI is the Received Signal Strength Indicator value.

The XBee RF Modules interface to a host device through a logic-level asynchronous serial port. Through its serial port, the module can communicate with any logic and voltage compatible UART. Devices that have a UART interface can connect directly to the pins of the RF module as shown in
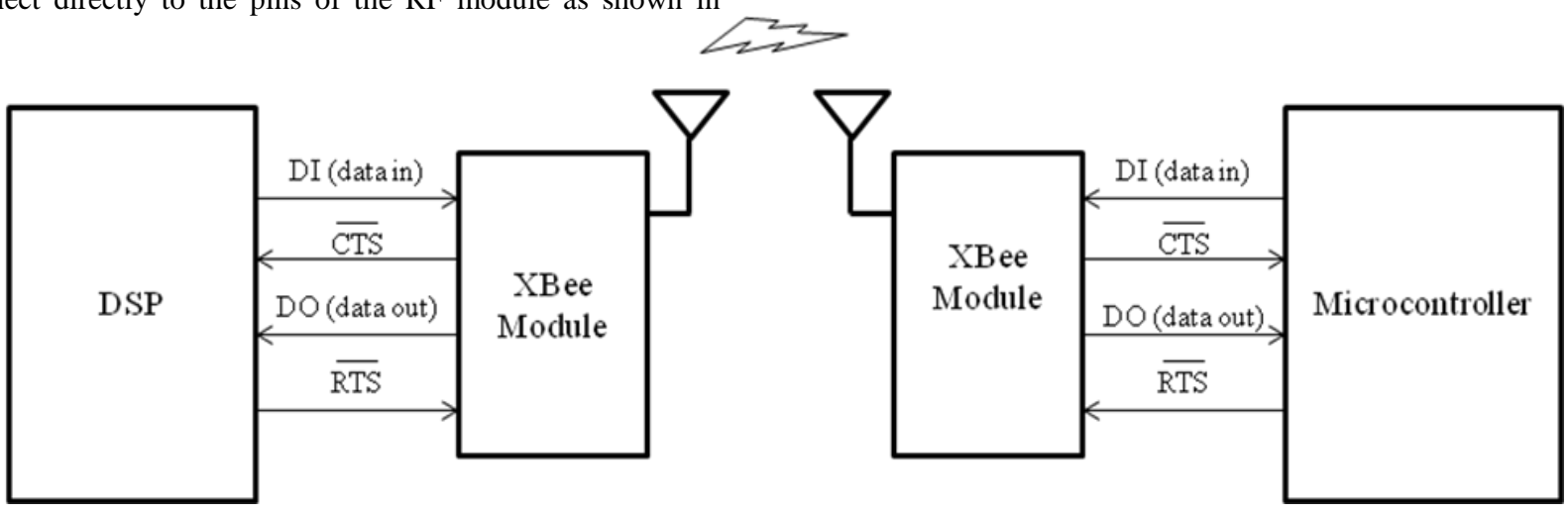

Fig 7: UART-interfaced environment

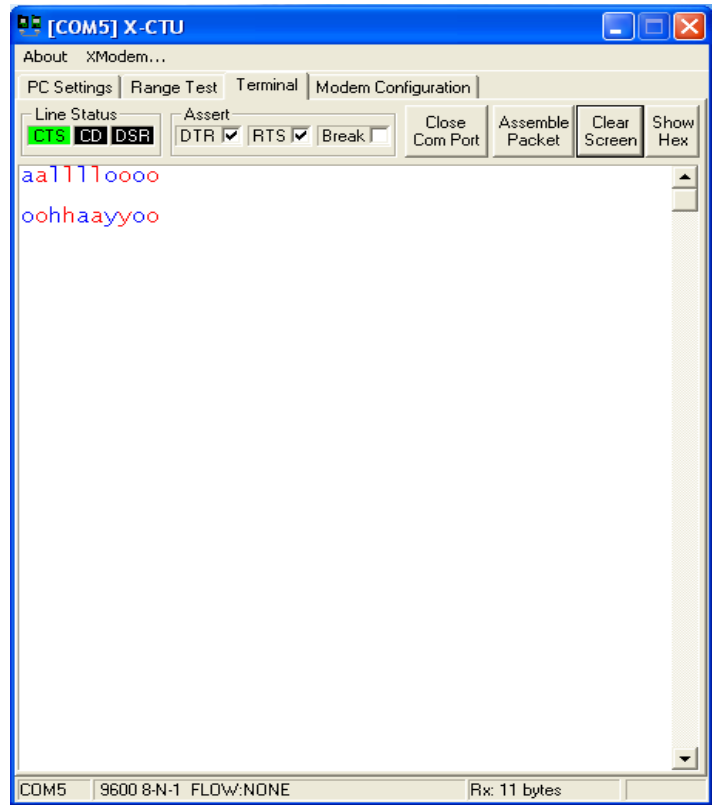

(a) Byte transmission

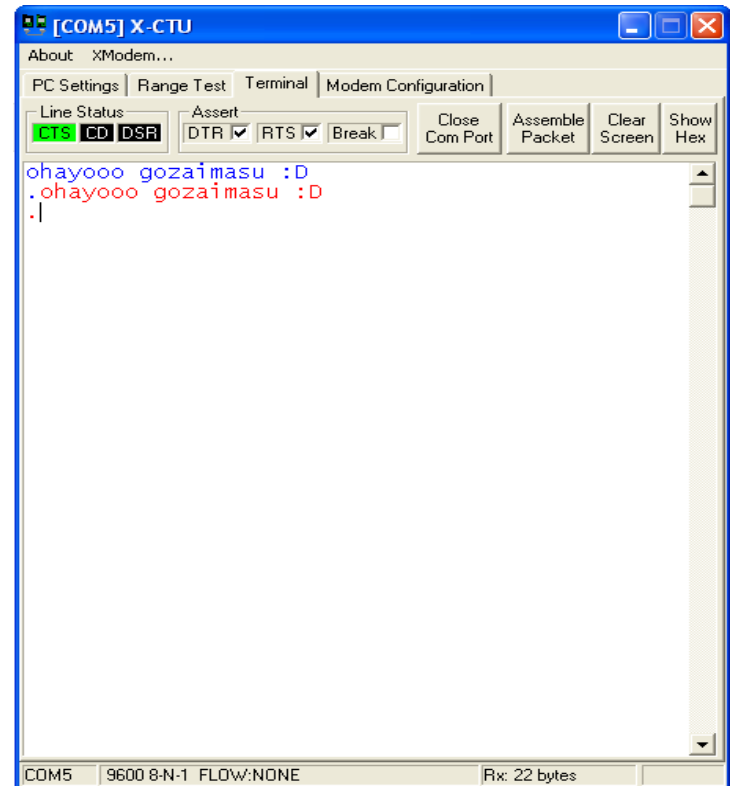

(b) Packet transmission

Fig 8: Loopback test 

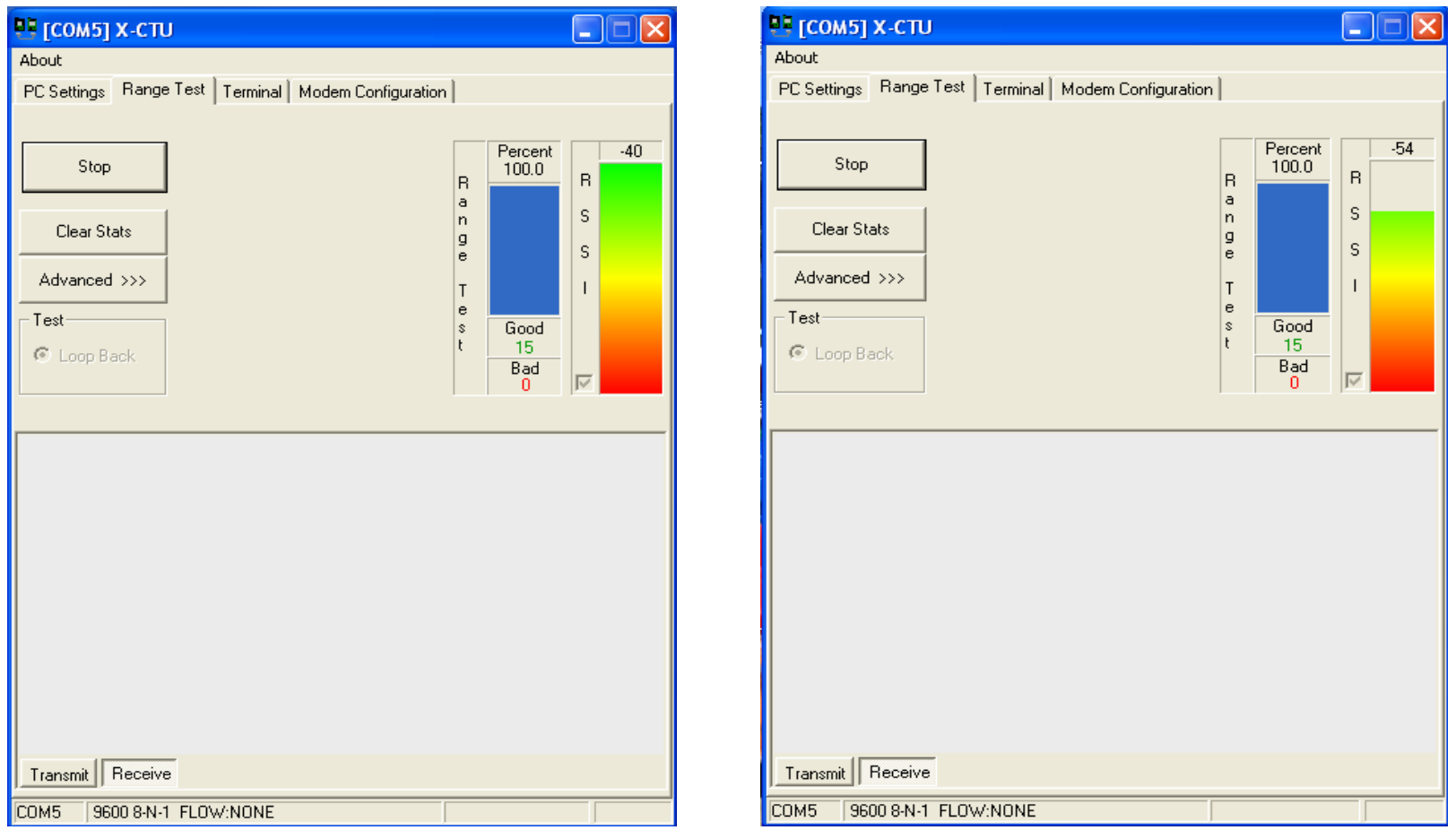

Fig 9: Range Test

\subsubsection{Control}

The transmitted control characters are received by the microcontroller and compared with some predefined characters. If there is a match, the microcontroller will switch the corresponding relay and turn on/off the appliance connected to it. The components of the control part are as shown in Figure 10. Figure 11 shows the microcontroller development board on which the ATmega8 is mounted.

The microcontroller used is ATmega8 from ATMEL. It consists of 3 ports namely an 8 -bit port $\mathrm{D}$, a 6 bit port $\mathrm{B}$ and another 6 bit port $\mathrm{C}$ and also has 2 pins for an external crystal oscillator as well as a reset pin. It has ADC pins multiplexed with port $\mathrm{C}$ and a 'receive' and 'transmit' pin at PD0 and PD1 respectively which are connected to the $\mathrm{XBee}$.

The relay circuit being used consists of a ULN2803 relay driver and $12 \mathrm{~V}$ relays which are used to operate the appliances. The relay driver is used to increase the current gain, so as to be able to switch the relays. Figure 12 shows how the relay is connected to the microcontroller.

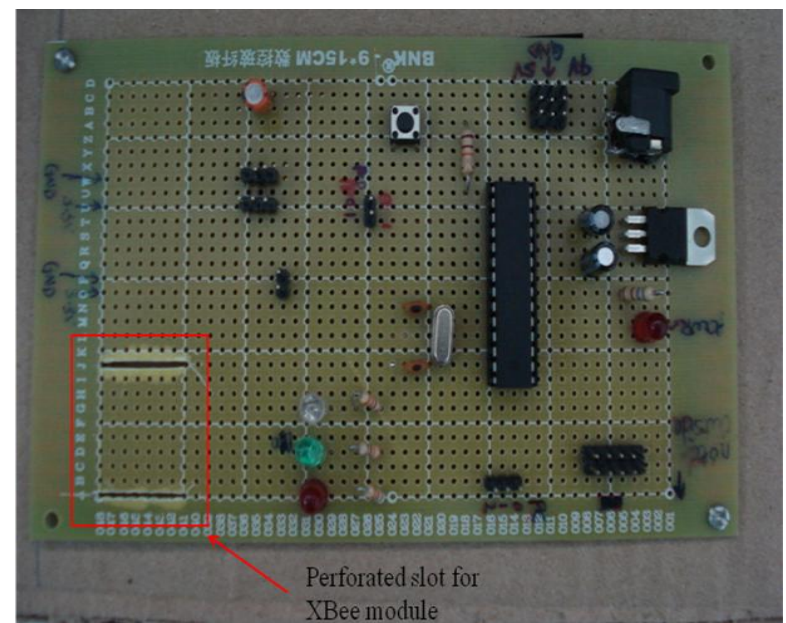

Fig 11. Microcontroller Development Board

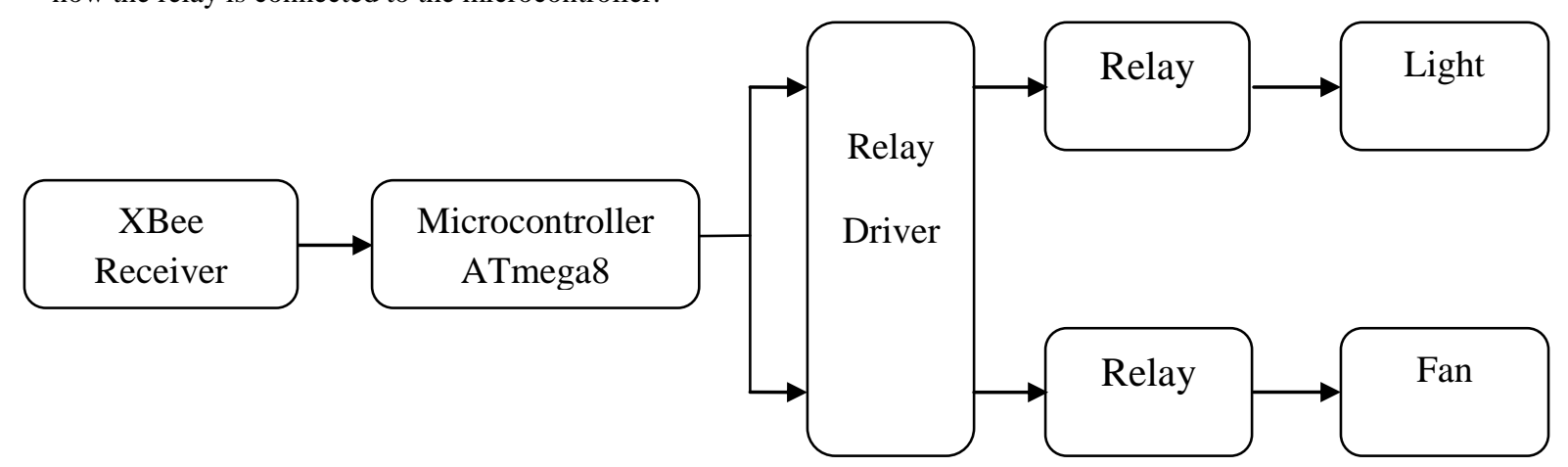

Fig 10: Block diagram of control part 


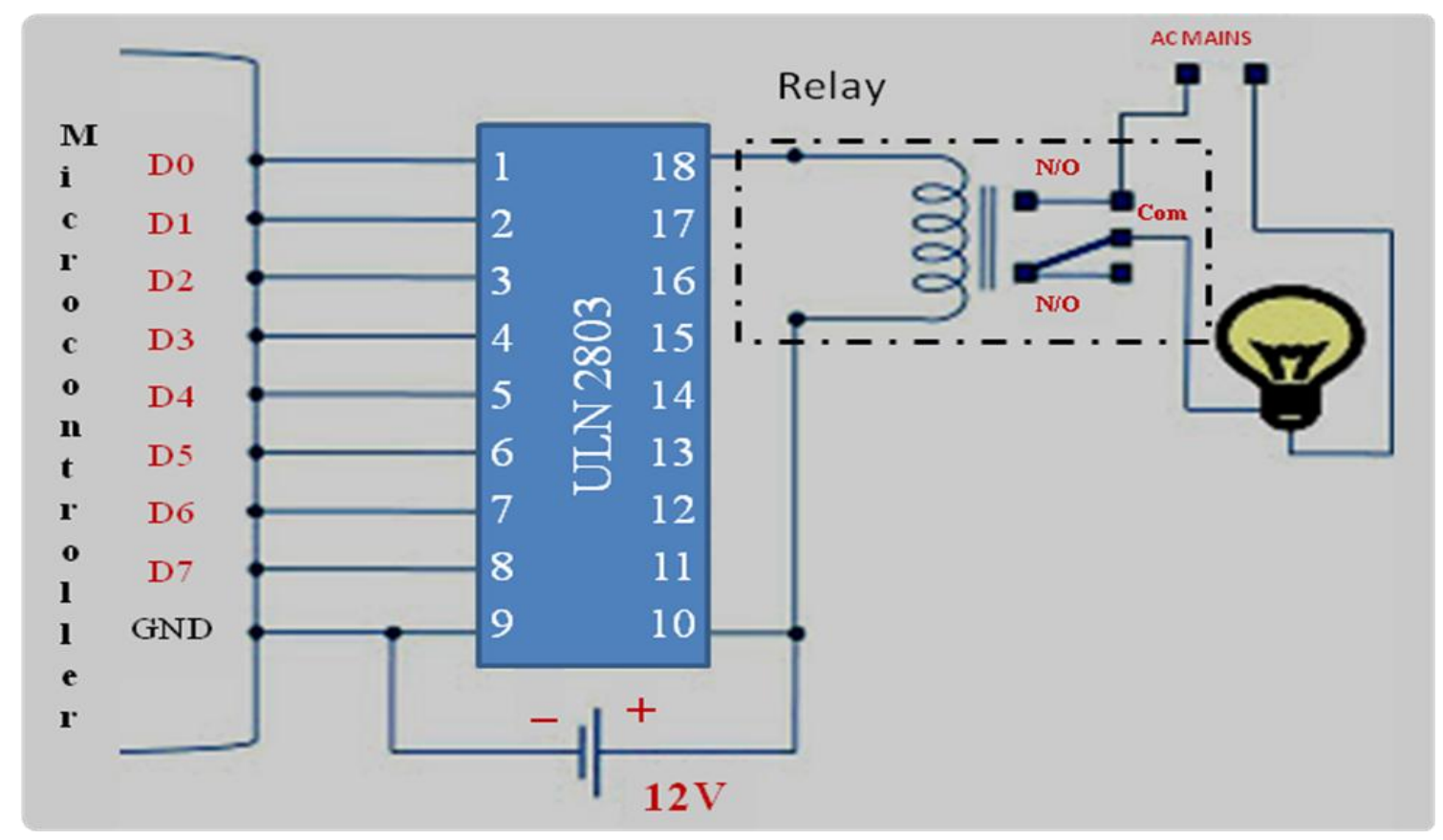

Fig 12: Connection of relay to microcontroller

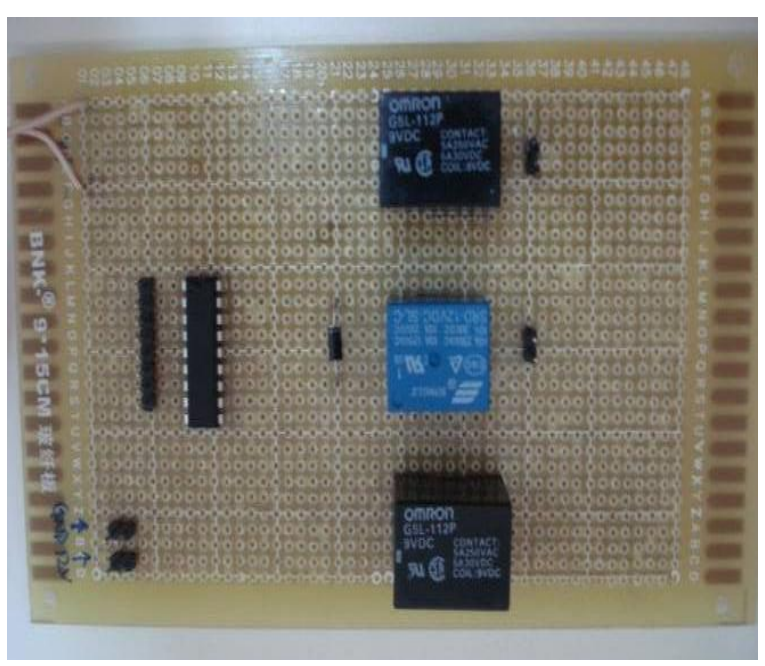

Fig 13: Relay board

\subsection{Software Design}

The Software design consists of the voice recognition section and programming of the control part.

\subsubsection{Voice recognition}

The Voice recognition is implemented using the DSP kit. The main program is written using $C$ language in Code Composer Studio which is an integrated development environment for Texas Instrument's embedded processors, being in this case the C5535 eZdsp kit. Voice recognition is performed using the ZCPA method, which is the simplest reliable technique that can be used for this application. This method basically counts the number of times the signal crosses zero, and hence can determine the approximate frequency of the signal. Here, the intensity information is incorporated in the zero crossing value using a logarithmic function. The difference in frequency helps the system in distinguishing between the different voice commands. Figure 14 shows the flow diagram of the DSP program used to perform voice recognition. The DSP is first initialized in order to set the clock frequency, initialize the codec etc. The audio samples are then read from the microphone through the codec. These samples are processed using a transform known as Daubechies wavelets which reduces the noise in the signal, making it clearer. Next, we have to determine whether a voice signal is actually present. For this purpose, a 'noise-gate' is used, which filters out low frequency noise signals. Thus, noise is replaced by silence, which helps us in effectively determining when an actual voice signal is present. The system then checks for the reception of a password. If it is valid, all samples that are beyond a certain threshold level are stored in a buffer until the voice signal is no longer present. A zero crossing function is then applied on the buffer to determine the approximate frequency of the signal.

\subsubsection{Control}

On the control side, the microcontroller has to be programmed to be able to receive control characters from the receiver $\mathrm{XBee}$ module and activate/control the required relays accordingly. The program is written in C using AVR Studio and the hex file is loaded into the ATmega8 microcontroller using the ProgISP programmer software. The flow diagram for reception and control is given in Figure 15. A specific port of the microcontroller is set as the output port. This port will be used for controlling the turning on and off of the relays. Global interrupts are then enabled and the UART is initialized, which involves baud rate being set to $9600 \mathrm{bps}$ and data frame format being given, that is, 1 stop bit, 8-bit data, no parity etc. The microcontroller then waits for an interrupt to occur. Whenever a control character is received, the Interrupt Service Routine (ISR) runs and those characters are compared with predetermined ones. For example, if character ' $a$ ' is received from DSP, the first pin of port $\mathrm{C}$ is set high or low; similarly, if character ' $b$ ' is received, the second pin of port $C$ is activated or deactivated accordingly. If any other characters are received, no action is taken. After having activated/deactivated the corresponding pins, the microcontroller again waits for the next character to be received. 


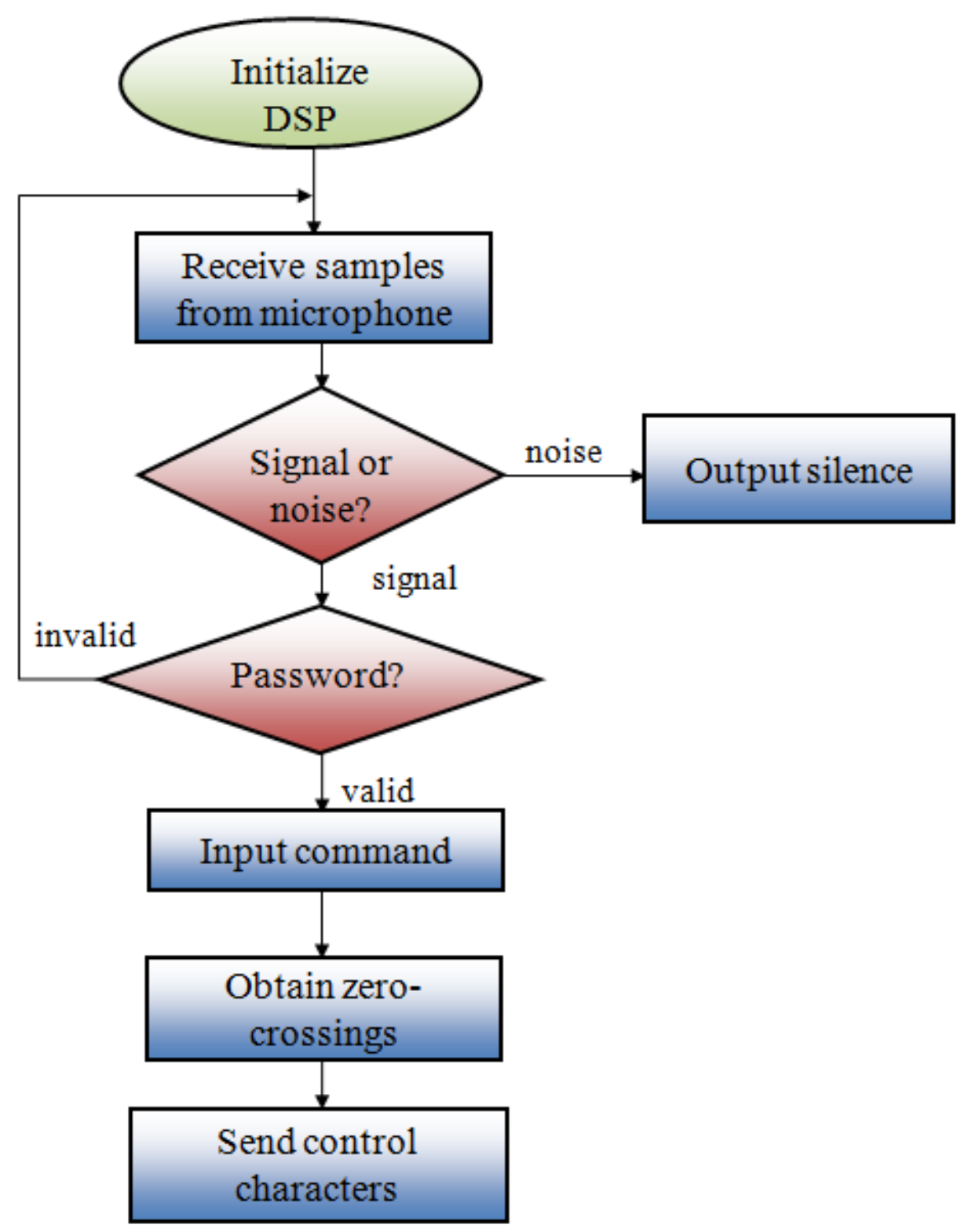

Fig 14: Flow diagram for voice recognition program

\section{RESULTS AND DISCUSSIONS}

The first step in this project was to write a program code so as to compute zero crossings with peak amplitude. This code was debugged using an integrated development environment known as Code Composer Studio. It was then loaded onto the DSP kit through the embedded JTAG emulator and run. Voice commands were then captured using a microphone connected to the DSP kit and processed to obtain zero crossing values. Corresponding control characters were then sent wirelessly through XBee modules to the microcontroller. The microcontroller used the received characters in order to activate corresponding output pins and consequently operate the home appliances through a relay driver and relay switches. Here, saying 'light' gives the control character ' $a$ ' to operate a light bulb and saying 'fan' gives ' $b$ ' which controls a DC fan. The complete system was implemented and tested by giving the 'light' and 'fan' commands to the DSP kit and observing how the control circuit responded. The Figure 16 shows the initial circuit indicating both the fan and bulb in the initial "off state". The user first gives the 'light' command to activate the light bulb. Figure 17 shows the result whereby the light bulb was successfully turned on by saying 'light'. 


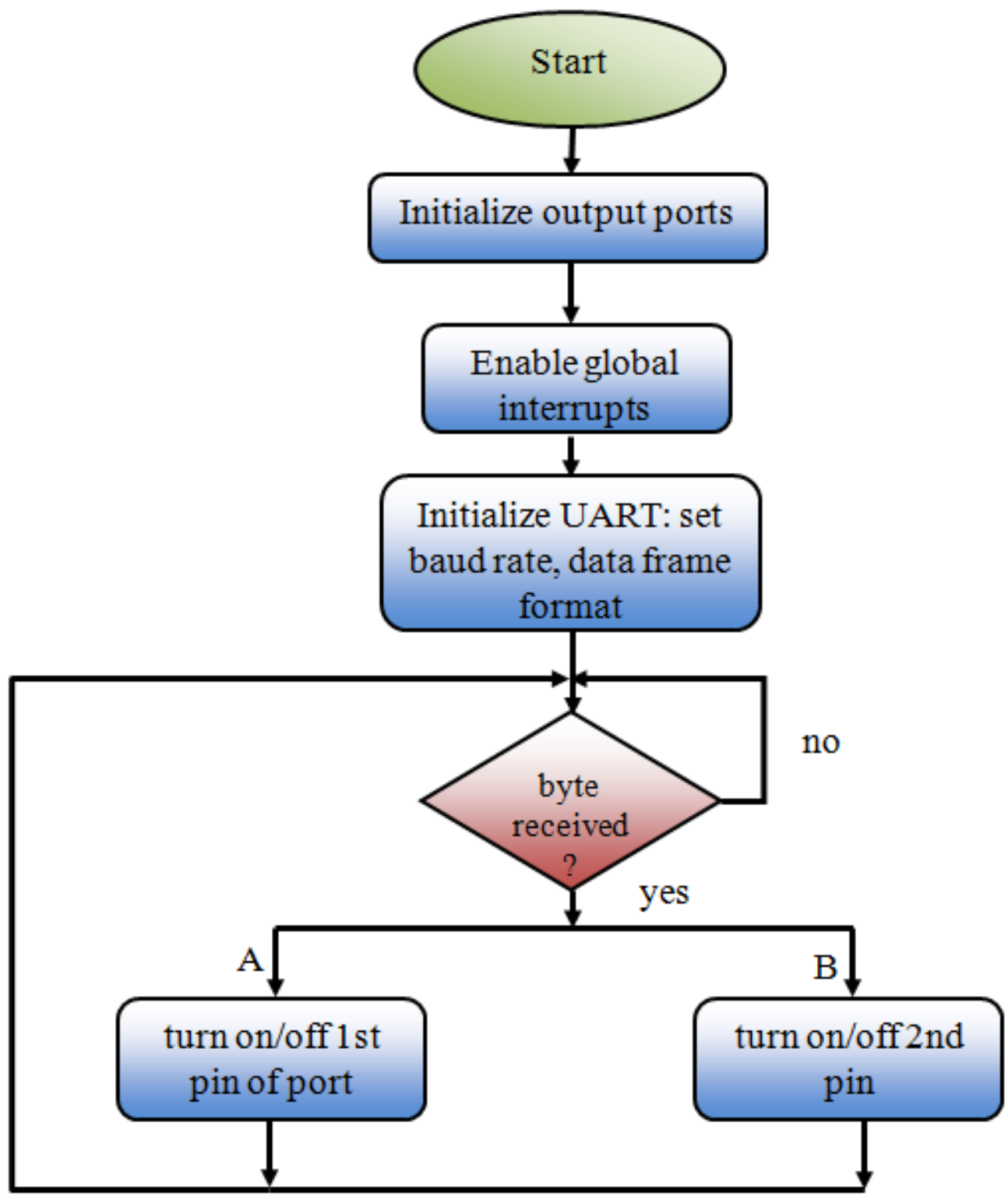

Fig 15: Flow diagram for reception and control by Microcontroller

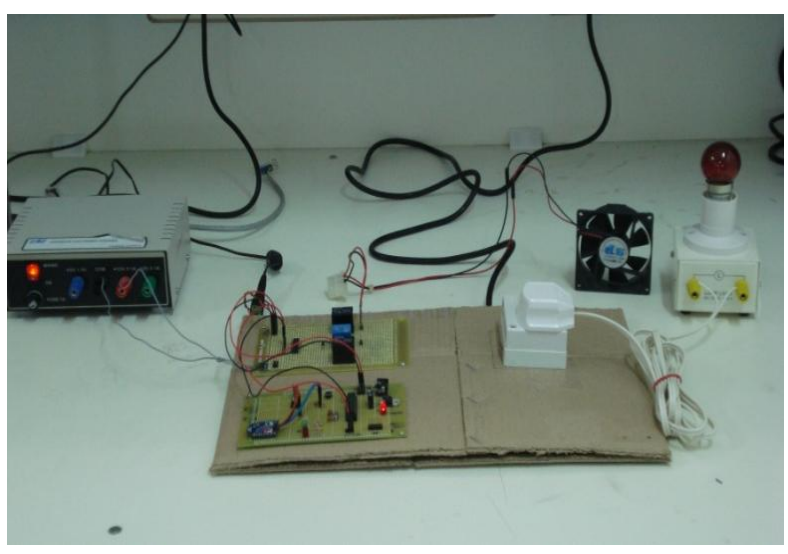

Fig 16: Initial state - Light off; Fan off

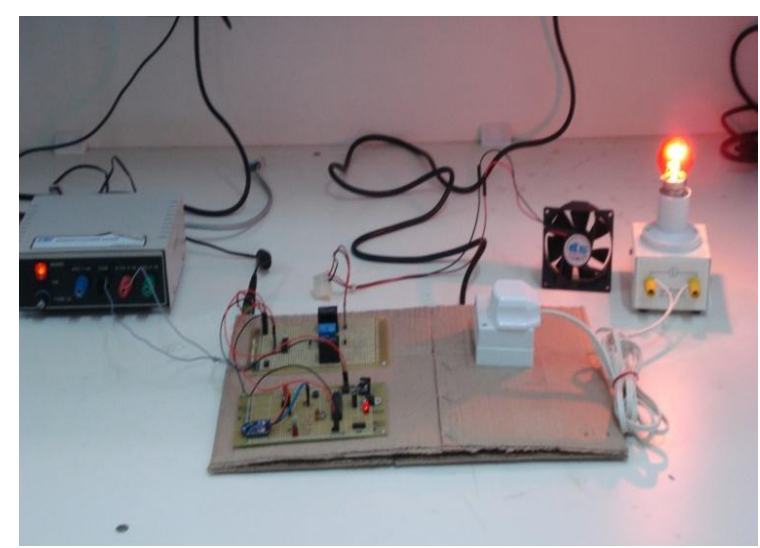

Fig 17: Light on; Fan off 
The 'fan' command is given next to activate the fan. Figure 18 shows the successful turning on of the fan while the light bulb remains in the on state when 'fan' is said.

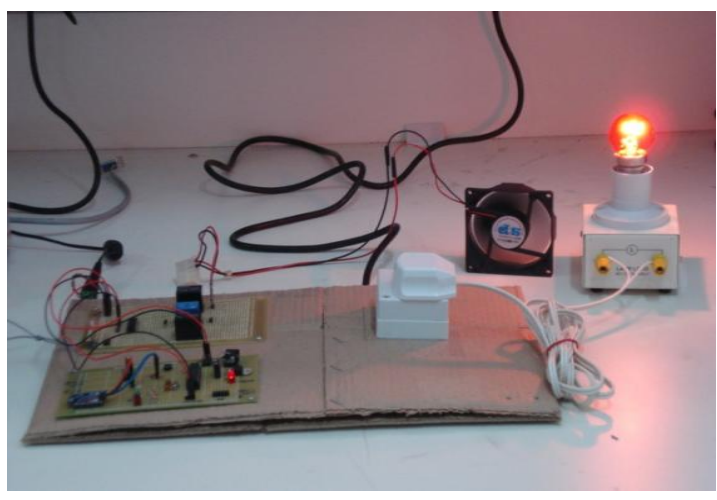

Fig 17: Fan on; Light remains on

Once both devices have been successfully switched on through the voice commands, the circuit is then tested for switching off both devices. When the light command is given for the second time, the light bulb switches off while the fan remains in the on state as shown in Figure 18.

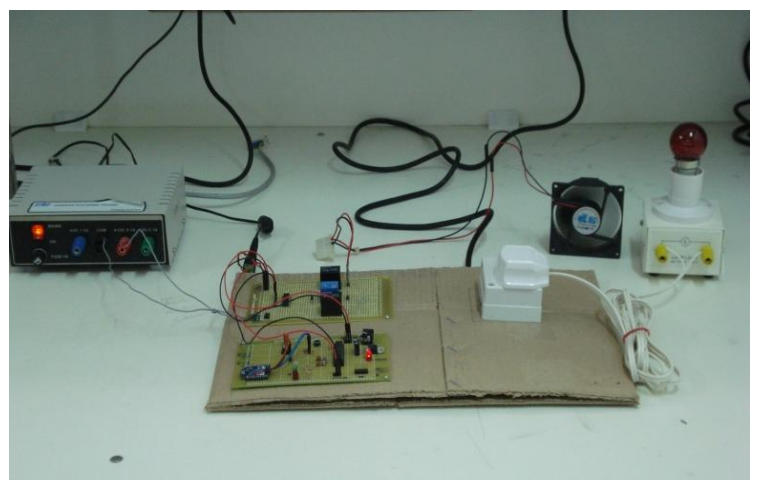

Fig 18: Light off; Fan remains on

Finally when the user says 'fan' for a second time, the fan will switch off as indicated by Figure 19.

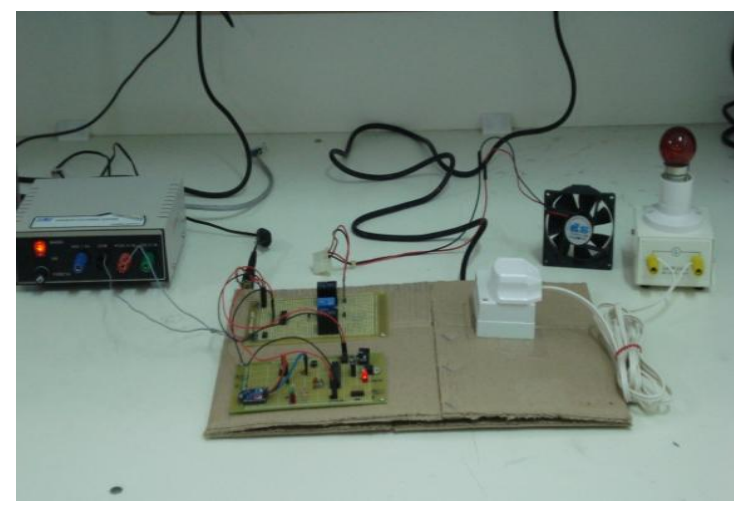

Fig 19: Light off; Fan off

We have thus been able to test and obtain favorable results using our Smart Home Control System Through Speech. The DSP recognized and successfully distinguished between the given voice commands. Therefore, the system was able to respond as expected and provided positive results. Figure shows the final system realization.

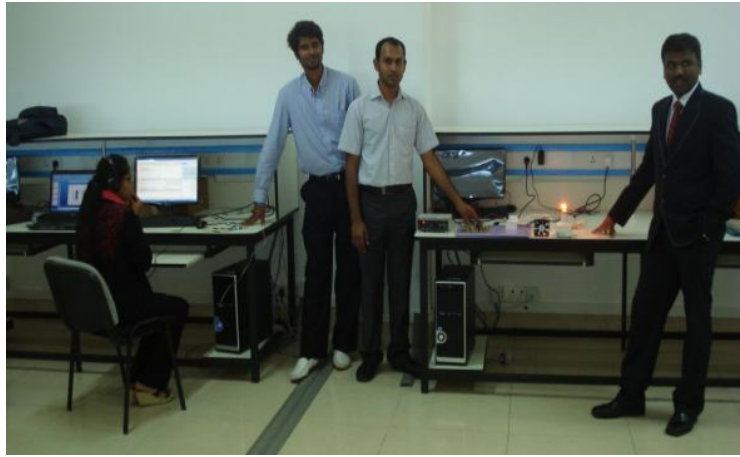

Fig 20: Final system realization

The proposed system developed can be used to control AC appliances through speech. This is cost effective and is highly accessible.

\section{CONCLUSION}

A home automation system based on voice recognition was built and implemented. The system is targeted at elderly and disabled people to help ease their life. The system developed can be used to control AC and DC appliances through speech. Voice recognition was successfully implemented using the low power TMS320C5535 DSP processor. The wireless communication was achieved using low powered XBee transceiver modules. Finally, power through appliances was controlled by making use of a microcontroller chip and relays. Hence we conclude that the aim of the proposed system has been attained and that the system is functioning as predicted. Through this system we have been able to control the switching on and switching off of two different devices solely through voice commands. The proposed system therefore provides solutions for the problems faced by old or disabled persons in daily life and makes their life easier and more comfortable by proposing a cost effective and reliable solution. Since low-powered components were used, this system proved to be power efficient, thereby saving energy.

\section{REFERENCES}

[1]. S. K. Hasnain, A. Beg and S. Awan, "Frequency Analysis of Urdu Spoken Numbers Using MATLAB and Simulink" Journal of Science \& Technology PAF KIET ISSN 1994- 862x, Karachi, Dec. 2007.

[2]. F.Wallam, M. Asif, "Dynamic Finger Movement Tracking and Voice Commands Based Smart Wheelchair", International Journal of Computer and Electrical Engineering, Vol. 3,No. 4, August 2011,pp.497502.

[3]. H. Chin, J.Kim, I.Kim, Y.Kwon, K.Lee, S. Yang, "Realization of Speech Recognition Using DSP (Digita1 Signal Processor)", IEEE International Symposium On Industrial Electronics Proceedings 2001, pp.508-512.

[4]. Y.Usha Devi, "Wireless Home Automation System Using ZigBee", Nalanda institute of Engg., and tech., Department of ECE, International Journal of Scientific and Engineering Research Volume 3, Issue 8, August2012

[5]. Tam Van Nguyen, Dong Gun Lee, Yong Ho Seol, Myung Hwan Yu, Deokjai Choi, "Ubiquitous Access to Home Appliance Control System using Infrared Ray and Power Line Communication", ICI 2007, $3^{\text {rd }}$ IEEE/IFIP 
International Conference in Central Asia, Tashkent, Uzbekistan, vol 1, pp1-4,26-28 Sept.2007

[6]. S. M. Anamul Haque, S. M. Kamruzzaman and Md. Ashraful Islam1 "A System for Smart-Home Control of Appliances Based on Timer and Speech Interaction" Proceedings of the 4th International Conference on Electrical Engineering \& $2^{\text {nd }}$ annual Paper Meet 26-28 , pp. 128-131,January, 2006

[7]. Malik Sikandar, Hayat Khiyal, Aihab Khan, and Erum Shehzadi "SMS Based Wireless Home Appliance Control System (HACS) for Automating Appliances and Security". Issue in Information Science and Information Technology Vol 6, Pp 887-894, 2009.

[8]. N.P.Jawarkar, Vasif Ahmed and R.D. Thakare. "Remote Control using Mobile through Spoken Commands". IEEE - International Consortium of Stem Cell Networks (ICSCN) 2007. 22-24, Pp.622-625, 2007.

[9]. Prabhakar V. Mhadse and Amol C.Wani "Speaker Identification Based Automation System Through Speech Recognition" in International Journal of Emerging Technology and Advanced Engineering, Volume 3, Issue 1, January 2013 pp 557-563.

[10].S. R. Suralkar, Amol C.Wani and, Prabhakar V. Mhadse, "Speech Recognized Automation System Using Speaker Identification through Wireless Communication" in IOSR Journal of Electronics and Communication Engineering, Volume 6, Issue 1,2013, pp 11-18.
[11].Texas Instruments C5535 eZdsp USB Stick Development Kit datasheet

\section{AUTHOR'S PROFILE}

Mr. Parameshachari B D is working as a Senior Lecturer in the Department of ECE at JSSATE, Mauritius. He is working at JSSATE, Mauritius since from July 2010 and worked as a Lecturer at Kalpatharu Institute of Technology, Tiptur for Seven years. He obtained his B.E in ECE and M. Tech in Digital communication engineering from Visvesvaraya Technological University, Belgaum, Karnataka, India. He is pursuing his Ph.D in ECE at Jain University, Bangalore, Karnataka, India. His area of interest and research include image processing and cryptography. He has published several Research papers in international Journals/conferences. He is a Member of ISTE, IETE, IACSIT, IAEST, IAENG and AIRCC.

Mr. Sawan Kumar Gopy is studying final year BE in Electronics and Communication Engineering at JSSATE, Mauritius, affiliated to Visvesvaraya Technological University, Belgaum.

Ms. Gooneshwaree Hurry is studying final year BE in Electronics and Communication Engineering at JSSATE, Mauritius, affiliated to Visvesvaraya Technological University, Belgaum.

Mr. Tulsirai T. Gopaul is studying final year B E in Electronics and Communication Engineering at JSSATE Mauritius, affiliated to Visvesvaraya Technological University, Belgaum. 